\title{
Software Quality Assurance (SQA) : Current and Emerging Trends
}

\author{
S Ravichandran ${ }^{1}$, Karthik Padmanabhan ${ }^{2}$, P. M. Shareef ${ }^{3}$ \\ ${ }^{1}$ CEO and Chief Scientist, Trimentus Technologies, ${ }^{2,3}$ Executive Vice President, Trimentus Technologies Pvt. Ltd. \\ $3^{\text {rd }}$ Floor, Sucons Sivagami Square, 147, G N Chetty Road T Nagar, Chennai - 600017 \\ Tele : $+91-9841016968$ \\ Website : www.trimentus.com
}

\begin{abstract}
The role of the Software Quality Assurance (SQA) within an organization has always been critical one. This is also a role which has seen a rapid evolution over the last decade or so. We have seen the SQA role move from being primarily responsible for process compliance to now being actively involved in strategic organizational governance. This technical paper charters the path of the SQA role within an organization commencing with explaining its traditional responsibilities and activities and culminates with what we believe would be the future focus of this extremely important role
\end{abstract}

Key Words : Software quality assurance. Process compliance

\section{SQA: A Few Definitions}

Table 1. Definitions

\begin{tabular}{|l|l|}
\hline Source & \multicolumn{1}{|c|}{ Definition } \\
\hline CMMI-Devv1.2 & $\begin{array}{l}\text { A planned and systematic means for assuring } \\
\text { management that the defined standards, practices, } \\
\text { procedures, and methods of the process are applied. }\end{array}$ \\
\hline NASA $^{1}$ & $\begin{array}{l}\text { Software Quality Assurance (SQA) is defined as a } \\
\text { planned and systematic approach to the evaluation } \\
\text { of the quality of and adherence to software product } \\
\text { standards, processes, and procedures. SQA } \\
\text { includes the process of assuring that standards and } \\
\text { procedures are established and are followed } \\
\text { throughout the software acquisition life cycle. }\end{array}$ \\
\hline IEEE & $\begin{array}{l}\text { Software quality assurance is: } \\
\text { 1. A planned and systematic pattern of all actions } \\
\text { necessary to provide adequate confidence that } \\
\text { an item or product conforms to established } \\
\text { technical requirements. }\end{array}$ \\
\cline { 2 - 3 } & $\begin{array}{l}\text { 2. A set of activities designed to evaluate the } \\
\text { process by which the products are developed or } \\
\text { manufactured. }\end{array}$ \\
\hline
\end{tabular}

All the definitions of SQA points (Refer Table1) to the same premise: The quality of a system is highly influenced by the quality of the process used to acquire, develop, and maintain it. This premise implies a focus on processes as well as on products. This is a long-established premise in manufacturing. Belief in this premise is visible worldwide in quality movements in manufacturing and service industries and this is applicable to software development and maintenance also.

Hence, the principal role of SQA members is to ensure that efficient and effective processes are defined and deployed within an organization which in turn ensures that good quality products are delivered on time and within budget.

\section{SQA GROUP'S RESPONSIBILITIES AND THEIR EVOLUTION}

The role of the SQA group is a constantly evolving one, where greater and larger responsibilities are being undertaken constantly. SQA responsibilities have evolved from that of a process caretaker, as used to exist earlier, to being a central catalyst for change now. The QA unit has become a strategic unit to imbibe a quality focused culture within the organization that will enhance the organization's ability to manage its processes and systems in such a way that it delivers significant cost, time and process efficiencies to its customers. Quality Management requires the ability to identify key processes within the organization that affect quality and manage these processes to deliver tangible benefits to the customer.

Overall, in the current IT scenario, the SQA group's primary KRAs can be summarized to be under 3 main areas:

1) Process compliance, implementation and institutionalization

\section{2) Process improvement}

3) Management visibility and increased responsibilities

Let us examine their role in each of these areas, and while doing so, also discuss their evolution.

\section{Process compliance, implementation and institutionalization}

\section{Process compliance}

This is the most traditional role of the SQA: To ensure compliance against the defined processes and standards.

Typically, Compliance with agreed-upon standards and procedures is evaluated through process monitoring 
and audits. Software development and control processes would include quality assurance approval points, where an SQA evaluation of the product may be done in relation to the applicable standards. Performing regular, periodic and event driven audits was the key responsibility in ensuring compliance.

In this case, the primary role of SQA personnel within the organization involves

- Objectively reviewing activities, work products, and services against applicable requirements, process description, standards, and procedures

- Identifying and documenting noncompliance issues

- Providing feedback to project staff and managers regarding the results of the quality assurance activities

- Ensuring that noncompliance issues are addressed

The key ingredient of this role was the objectivity that the group bought in. SQA were involved in reviewing activities and work products against defined criteria that minimized subjectivity and bias by the reviewer. Those performing quality assurance activities would be separate from those directly involved in developing or maintaining the work products. An independent reporting channel to the appropriate level that organizational management allows would be available so that noncompliance issues may be escalated as necessary.

Noncompliance issues are problems identified in audits that reflect a lack of adherence to applicable standards, process descriptions, or procedures. When local resolution cannot be obtained, established escalation mechanisms are used to ensure that the appropriate level of management can resolve the issue.

\section{Process implementation and institutionalization}

Implementation of the standard/ defined processes is a slight evolution from the previous role. SQA undertook the increased responsibility where members acted as facilitators in explaining key processes and usage to the practitioners. Quality assurance activities typically were initiated in the early phases of a project to establish plans, processes, standards, and procedures that will add value to the project and satisfy the requirements of the project and the organizational policies. SQA members facilitated process tailoring and customizations being performed for a project. Those performing quality assurance participate in establishing the plans, processes, standards, and procedures to ensure that they fit the project's needs and that they will be useable for performing quality assurance evaluations.
The SQA also began to be involved in all critical project meetings such as Project Initiation meeting, kick off meeting, End Phase meeting and Close out meetings to ensure that relevant agenda points are addressed. Facilitation was a key aspect of the SQA functioning. Project Managers were advised during preparation of all plans; Project team members were guided on all quality related aspects in the project.

SQA members were also involved in providing training on quality systems and processes to all project members. Process training, often, was a key enabler in ensuring that processes are implemented and institutionalized consistently across the organization.

\section{Process improvement}

One of the most significant trends that emerged from implementation of models such as CMM and CMMI were creation of specific groups focused on process improvement such as the SEPG and Process Owner groups. More importantly, these groups were either amalgamated with the SQA or had a direct correlation to each other.

The SEPG had the direct responsibility of coordinating and effecting process improvements within an organization.

The SQA now became the most important source for process improvements and acted as a direct feeder for process changes to the SEPG. Since the SQA, as a virtue of their process compliance, implementation and institutionalization role had literally a daily, hands-on interaction with the practitioners, were acutely aware of the process inefficiencies and bottlenecks in the standard process. The SQA was ideally positioned to be cognizant of the process behaviour, had complete understanding of project problems and its attribution to various processes and subprocesses.

This understanding translated, therefore, as inputs to the SEPG to identify process changes and improvements. Nearly $75 \%$ of the process improvements identified have been sourced from the SQA in any given organization.

Nowadays, the SEPG and SQA have been so closely amalgamated that SQA group behaves almost like a subset of the overall SEPG unit. Most organizations empower SQA members to undertake several process improvement initiatives. In several organizations, all members of the QADept. are members of process groups even including the Tools Group, whose key responsibilities are to develop methods, tools and processes that facilitate and ensure that standards are adhered to and specifications are met and identify and develop tools that facilitate process implementation, improve engineering as 
well as support processes, improve productivity etc.

The SQA units are increasingly involved in even carrying out core engineering activities in order to:

- Optimize software engineering practices

- Investigating techniques for reducing software development and maintenance costs while simultaneously improving the quality of the systems produced

- Develop techniques, tools and methods to support process implementation

- Reduce and prevent defects through innovative studies (such as Fault Injection modelling, FMEAetc)

Software engineering activities carried out by QA units include development and improvement of process models, defect models, data analysis and other engineering activities.

The impact of SQA activities are directly measured through a quantitative index named, Process Compliance Index (PCI). The relation between Process Compliance Index (PCl) (internal parameter) and Customer Satisfaction Index (CSI) (external parameter) was extensive studied. These studies reveal the following trend. (Refer Fig.1)

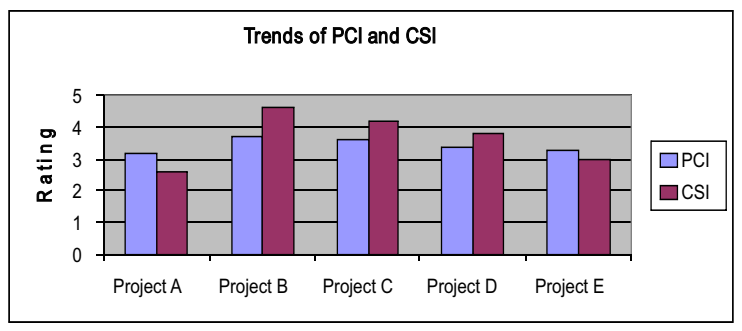

Fig. 1. Trends of PCl and CSI

To understand the statistical significance of the relation, a regression analysis was done. By carrying out regression analysis a straight line equation was arrived at for predicting customer satisfaction index based on the existing process compliance index. While the empirical equation derived gives a direct indication of higher process compliance resulting in greater customer satisfaction, more data on customer satisfaction needs to be considered to improve the accuracy of the equation to predict the customer satisfaction upfront. Fig. 2.

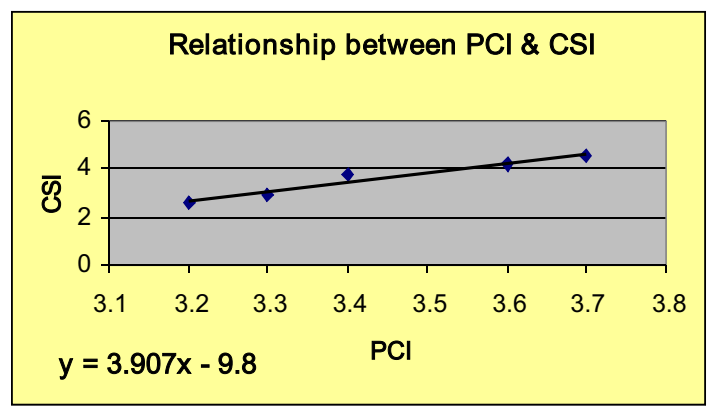

Fig. 2. Relationship between PCI \& CSI

- Typical straight line equation $y=m x+c$ where, $y$ is dependent variable (CSI), $x$ is independent variable (PCl)

- $m$ is the slope of the line (indicates increase in y per unit increase in $\mathrm{x}$ ) and $\mathrm{c}$ is the intercept point at which straight line cuts $y$-Axis i.e. value of $y$ when $x=0$.

The regression analysis carried out for the independent variable $(\mathrm{PCl})$ and dependent variable (CSI) indicates good linear relationship between the two. The straight-line equation derived based on data available will be of use to predict the impact of process compliance index on customer satisfaction index.

\section{Management visibility and increased responsibilities}

Finally, the SQA responsibilities have grown to the extent of them being the 'eyes and ears' of the Management. One of the main objectives of the SQA is to provide staff and management with objective insight into processes and work products.

SQA Members are involved from project start-up onwards in facilitate Project Managers in preparation of all project management plans. Reviews of most plans are undertaken by the SQA members to ensure that all planning parameters are planned effectively.

Increasingly, the role of the SQA in project meetings is being greatly enhanced. SQA in now involved to perform reviews and analysis of metrics using statistical and management tools and undertake subsequent corrective and preventive actions. In most organizations, the SQA is now allocated the responsibility to independently monitor key project parameters including schedule, effort and size variances, defects identified during the verification/ validation activities, risk materialization etc and report them to the Senior Management.

There have been numerous instances of SQA members moving laterally to become highly successful Project Managers due to their propensity to sound project management techniques. There are also instances of the 
reverse: PMs enjoying very successful careers as SQA Leads and analysts.

\section{What the SQA is not}

In several cases, the role of the SQA has been confused with that of Verification and Validation activities. 'QA' is often likened to the testing role, especially in the US. The SQA practices ensure that planned processes are implemented, while Verification/Validation processes ensure that the specified requirements are satisfied. These two areas may on occasion address the same work product but from different perspectives. It is important to note the differences and understand the role of SQA correctly.

\section{Benefits to the organization}

The results of the performance of a SQA unit can be tangibly seen and measured across multiple parameters. To name a few, the results and benefits can be seen across:

- Organizations Business Results

- Client Satisfaction Index and its Trend

- Accuracy of Early Warning Signals

- Number of 'Red' Projects

- Accuracy of Software and Operational Metrics

- Efficiency and effectiveness of Quality System

- Overall process compliance

\section{Current challenges for SQA}

Of course, the SQA path is not without some obvious challenges.

\section{Plethora of quality models}

Currently, there are too many best practice Quality Models/ Frameworks (ISO, CMMI, COPC, eSCM, PCMM, ITIL, etc.) resulting in duplication of effort and discordant Quality systems. While each model has its area of application, there are overlaps and several organizations are into what may be termed as "model mania". It would be impossible for a SQA member to be well versed in all these models and to interpret and implement them adequately.

\section{An explicit understanding of a career path}

While there is an excellent growth path in the SQA field, this career path is not visible or not understood well by aspiring professionals and hence this area only becomes a secondary or a tertiary choice when choosing a career. Hence the industry has not been able to attract and retain the best professionals, in required numbers, in the SQAdomain

\section{Gap to the academia}

While core software engineering skills has now become an integral part of the academia, and almost universities incorporate software engineering as a specialized subject, SQA has remained outside the academic curriculum.

\section{III - MOVING FORWARD: WHAT IS NEXT IN STORE FOR THE SQA}

With the increased involvement of the SQA in project activities, defining organizational processes, and overall organizational governance, the future is bright for the SQA role. The following are what we believe, to be the immediate focus of SQA members in the near future:

The software development scenario is moving ahead with several technologies, environments, and platforms being launched in an incredible pace. These includes virtual appliances, virtual offices, SOA, SaaS, P2P monitoring systems, Event driven architectures etc. The current processes and indeed the current crop of SQA members will have to be quickly acquainted with these emerging trends to remain productive. But this in turn offers wonderful opportunities for SQA members to learn and adapt to changing environments and even be the catalyst for change within the organization. They play a crucial role in creating processes for seamless adoption and usage of these technologies.

Workflow and Automation of QMS at large offer huge potential for improvement and an integrated Project Management Tool is on every organizations process agenda. The role of SQA in these initiatives is immense in enabling these changes to happen within an organization.

With an increased focus on high maturity process areas with the associated scrutiny into it, it is becoming mandatory that a thorough statistical knowledge be an essential part of the SQA skill set.

Amidst the implementation of all the various process models, the strident voice of the Project Managers highlighting the overheads and costs involved in using heavy processes are also being heard. It is a fact that processes are becoming unwieldy and cumbersome to use as a result of QMS being the subject of several models. The industry is awakening to this fact and hence demanding SQAs to create leaner, simpler and at the same time effective processes. The Lean methodology offers promise which is still untapped in the IT field.

\section{CONCLUSION}

We have seen that the SQA role has evolved considerably from being primarily responsible only for process compliance to now being actively involved in 
strategic organizational governance. This does not mean that the traditional roles of the SQA, such as process compliance, have been diluted. Conversely, the portfolio of a SQA member has only been greatly enhanced to include challenging activities such as effecting process innovations, strategic management and being the driver for organizations to adopt new industry trends.

The impressive growth of the Indian IT industry reflects the confidence the world has in us and Quality is increasingly becoming the vital differentiator in a global and highly competitive market-place

We believe that the SQA has a very important role to play in the future growth of every IT organization.

\section{REFERENCES}

[1] Gartner's Emerging Trends, from http://www.adtmag.com/

[2] Software Assurance Technology Center (SATC), http://satc.gsfc.nasa.gov/

[3] Top 10 Emerging Software Development Trends for 2006, http://www.manageability.org/

[4] IEEE Definitions, http://ieeexplore.ieee.org/

[5] Anand Vaishampayan, Sept 2007, "Quality of Quality" presentation, IBM India, NASSCOM Quality Summit.

[6] Karthik Padmanabhan and Raghu R, 2003, "Customer delight through mature software process compliance" Technical paper SSI Technologies. 